\title{
RotoStep: A Chromosome Dynamics Simulator Reveals Mechanisms of Loop Extrusion
}

\author{
Josh Lawrimore, Brandon Friedman, Ayush Doshi, and Kerry Bloom \\ Department of Biology, University of North Carolina at Chapel Hill, North Carolina 27599-3280 \\ Correspondence: kerry_bloom@unc.edu
}

\begin{abstract}
ChromoShake is a three-dimensional simulator designed to explore the range of configurational states a chromosome can adopt based on thermodynamic fluctuations of the polymer chain. Here, we refine ChromoShake to generate dynamic simulations of a DNA-based motor protein such as condensin walking along the chromatin substrate. We model walking as a rotation of DNAbinding heat-repeat proteins around one another. The simulation is applied to several configurations of DNA to reveal the consequences of mechanical stepping on taut chromatin under tension versus loop extrusion on single-tethered, floppy chromatin substrates. These simulations provide testable hypotheses for condensin and other DNA-based motors functioning along interphase chromosomes. Our model reveals a novel mechanism for condensin enrichment in the pericentromeric region of mitotic chromosomes. Increased condensin dwell time at centromeres results in a high density of pericentric loops that in turn provide substrate for additional condensin.
\end{abstract}

There has been a revolution in understanding the higher-order structure and organization of chromosome in the past decade. Several major approaches (3C, ChromEMT, and super-resolution microscopy) are indicative of a disordered array of loopy fibers that emanate from an axial core (Dostie and Bickmore 2012; Dekker et al. 2013; Ou et al. 2017). The hierarchical models of structural intermediates building from 11 to $30 \mathrm{~nm}$ and larger fibers are not borne out in these recent 3D and live-cell studies $(\mathrm{Ou}$ et al. 2017). DNA looping was first observed in squash preparations of salamander eggs under the light microscope by the embryologist Oskar Hertwig in the early 1900s (Hertwig 1906). Paulson and Laemmli (1977) observed DNA loops when examining chromosome spreads in isolated mammalian cells. In metaphase, the loops emanate from a protein-rich chromosome scaffold. The chromosome scaffold is enriched in topology-adjusting proteins, such as topoisomerase II and the SMC (structural maintenance of chromosomes) proteins, known as condensin (Earnshaw et al. 1985; Hirano 2006).

Loops are a natural consequence of the entropic fluctuations and excluded volume interactions of tethered polymer chains in a confined space, such as the nucleus (Vasquez et al. 2016). If we consider the genome as a ball of yarn, the formation of loops can be appreciated as chains that randomly collide and wiggle around one another. Energy-requiring processes are also involved in loop formation. The earliest suggestion of loop extrusion came from the trombone model of DNA replication (Sinha et al. 1980; Alberts et al. 1983) and direct visualization of DNA looping at the replication fork (Park et al. 1998). More recently, SMC proteins (e.g., cohesin and condensin), which bind and hydrolyze ATP, have been cited as having loop extrusion potential (Alipour and Marko
2012). Condensin has garnered attention based on recent studies showing it to be a DNA translocase (Terekawa et al. 2017).

Condensin is composed of five subunits, two coiledcoils SMC2 and 4, a kleisin (Brn1), and two heat-repeatcontaining proteins (Ycs4 and $\mathrm{Ycg} 1)$. The heat-repeat proteins are likely to be sites of DNA-binding within the condensin complex. Terekawa et al. (2017) showed the ability of condensin to move processively along linear DNA sheets (Fazio et al. 2008). The challenge ahead is to understand, first, how local motion and topological constraints of the DNA exerted by condensin will be dissipated along the length of a long-chain polymer and, second, how the fluctuating, loopy genome feeds back to the ability of condensin to translocate processively.

ChromoShake is a statistical mechanics model depicting the motions of arrays of bead-springs (DNA) that show Brownian dynamics in a viscous environment (Lawrimore et al. 2016). The simulation has been applied to the centromere in budding yeast to reveal how the density of pericentromeric loops stiffen centromeric chromatin, imparting an active function to the centromere in mitosis (Lawrimore et al. 2016). In previous studies condensin was implemented as static springs (Lawrimore et al. 2016). Here, we introduce a new model, RotoStep, as a first-principles statistical mechanics approach to simulate condensin dynamics. Terekawa et al. (2017) have recently examined the behavior of single condensin molecules on DNA sheets. Terekawa et al. (2017) provides critical experimental metrics for evaluating results from simulation. Using RotoStep to simulate hand-over-hand motion (e.g., microtubule-based kinesin motor [Kull et al. 1996]), our simulations indicate that condensin can translocate along taut linear DNA and compact singly tethered DNA chains.

(C) 2017 Lawrimore et al. This article is distributed under the terms of the Creative Commons Attribution-NonCommercial License, which permits reuse and redistribution, except for commercial purposes, provided that the original author and source are credited. 
The dynamics of condensin stepping along single-tethered DNA result in extrusion of DNA loops. The same parameters for motor stepping result in drastically different geometries that are dictated by chromatin substrate dynamics. These simulations provide the first glimpse of how loop extrusion might work in living organisms.

\section{RESULTS}

\section{Model Assumptions}

Condensin is a DNA-based motor protein with the ability to translocate along double-stranded DNA at relatively high velocities (60 bp/sec) (Terekawa et al. 2017). To simulate condensin tracking DNA we used the array of bead-springs to depict both the DNA (long chain linear bead-spring configuration) and condensin (bead-spring chain). There are 11 beads in the condensin holocomplex; each bead is $\sim 10 \mathrm{~nm}$ in diameter. Condensin is a very flexible molecule, showing a persistence length of $\sim 4$ $\mathrm{nm}$ (Eeftens et al. 2016), about one-tenth that of DNA $(50 \mathrm{~nm})$. The chains of the antiparallel coiled-coils fold back on one another to length of $45 \mathrm{~nm}$. The complex adopts a number of configurations, including circular, Vshaped, and globular. The SMC protein coiled-coils are represented by beads $2-8$. To achieve DNA binding, we assert that the heat-repeat proteins (Ycs4, Ycg1) bind DNA (Piazza et al. 2014). These are beads 1 (representing Ycg1, red in Fig. 1) and beads 9 and 10 (representing Ycs4, white and red in Fig. 1) in simulations. The kleisin (Brn1) is represented as bead 11 (pink, Fig. 1) between the heat-repeat proteins connected via springs (not shown). This feature provides mechanical linkage between the leading and trailing points of contact.

The RotoStep program parses the coordinates, spring attachments, and indices of all beads in a ChromoShake simulation model. The binding mechanics of condensin changes when the condensin molecule becomes extended. We consider condensin-DNA binding unstable if the distance between beads 2 and 8 is $>30 \mathrm{~nm}$. If the distance between beads 2 and 8 is $<30 \mathrm{~nm}$ (Fig. 1A), the program determines the distances of the Ycs4 beads, 9 and 10 (rightmost white and red beads, Fig. 1A), to the Ycg1 bead, 1 (leftmost red bead, Fig. 1A). The closest Ycs4 bead to the Ycg1 bead is labeled proximal, and the other distal. A vector is drawn from the center of the proximal Ycs4 bead to the center of the distal Ycs4 bead and extended $10 \mathrm{~nm}$. The proximal Ycs4 bead is bound to the closest DNA bead to this point in space as the previous proximal Ycs4 bead DNA attachment is removed. This results in condensin stepping along the substrate resulting in loop extrusion (Fig. 1B).

If the distance between beads 2 and 8 is $>30 \mathrm{~nm}$ (Fig. 1C), the spring constant of the spring connecting the Ycg1 bead to the DNA is weakened 1000-fold. ChromoShake is then run to introduce thermal noise, allowing the springs between kleisin and beads 2 and 8, to pinch the condensin molecule together (Fig. 1D). On the next iteration of RotoStep, the Ycg1 bead is joined to the nearest DNA bead with a spring as the previous weak spring is simultaneously deleted. This results in the destabilization of the previous condensin-mediated loop and causes the Ycg1 end of condensin to bind DNA at a new location. After every iteration of RotoStep, ChromoShake is run on simulations to input thermal noise. The springs and kleisin-based threshold (30 $\mathrm{nm})$ are set to yield processive motion on a doubly tethered substrate (Fig. 2A), as described in Terekawa et al. (2017).

\section{Consequences of Processive Motion on Tethered Substrate with cis- and trans-Binding}

We set our condensin parameters (see Box 1) such that a single condensin could processively walk on an extended and pinned DNA substrate with a translocation speed of $60 \mathrm{bp} / \mathrm{sec}$ as reported in Terekawa et al. (2017). As shown in Figure 2A and Fig. 3A-C, upon loading condensin at one end, it processively migrates to the other end of the polymer chain.

Terekawa et al. (2017) reported a singly pinned DNA substrate incubated with condensin resulted in compacted DNA. We confirmed that our RotoStep simulation caused a singly tethered strand to compact (Fig. 2B,C and Fig. 3D, E). We added a single condensin to the tethered end of a singly tethered strand (Fig. 3D,E). Given that the kleisin spring never becomes taut, the strand is extruded as a loop. After several steps, a loop is spontaneously extruded as the strand adopts a random coil. Despite the random orientation
A

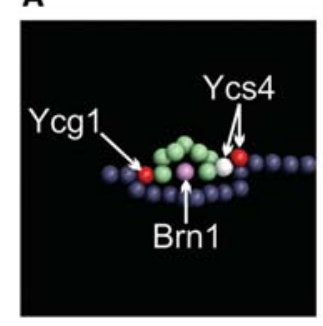

B

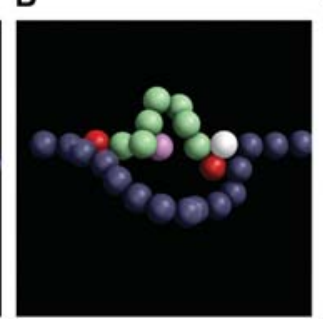

C

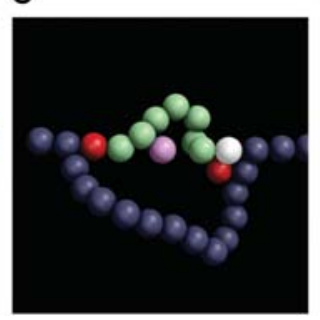

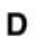

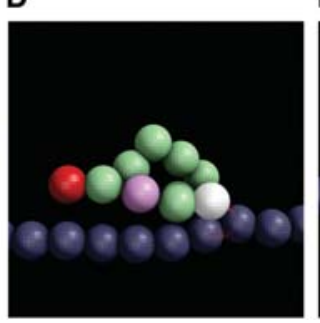

E

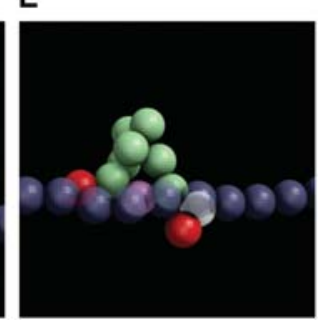

Figure 1. Simulated step and release algorithm. (A) Coiled-coil $\alpha$-helices of SMC proteins $(\mathrm{SMC} 2,4)$ are in green. Heat-repeat proteins Ycg1 (left red bead) and Ycs4 (white and right red beads) are bound to DNA (purple beads). The kleisin, Brn1 (pink bead), bridges the SMC containing subunits. $(B)$ One heat-repeat in Ycs4 (white bead) detaches and rebinds DNA depending on the projection of vector $(10 \mathrm{~nm})$ between the two heat repeats in Ycs4 relative to Ycg1 (see text). $(C)$ When springs linking kleisin (pink bead) to beads 2 and 8 in the SMC coiled-coils are extended $\sim 30 \mathrm{~nm}$, Ycg1 (trailing red bead) is released (shown in D). (E) Rebinding of Ycg1 to DNA following kleisin spring recoil. This results in directed motion on a taut chain. 
A
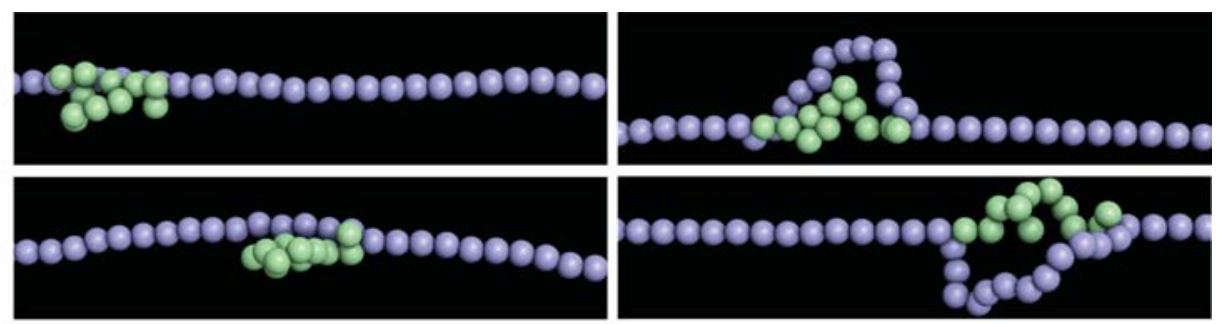

B
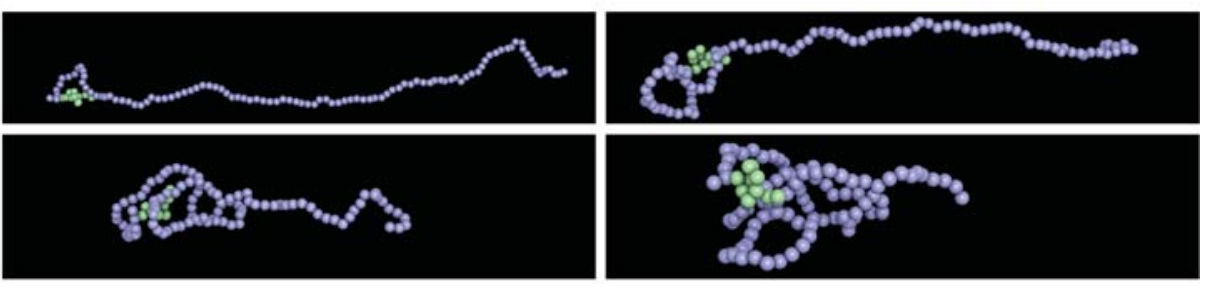

C
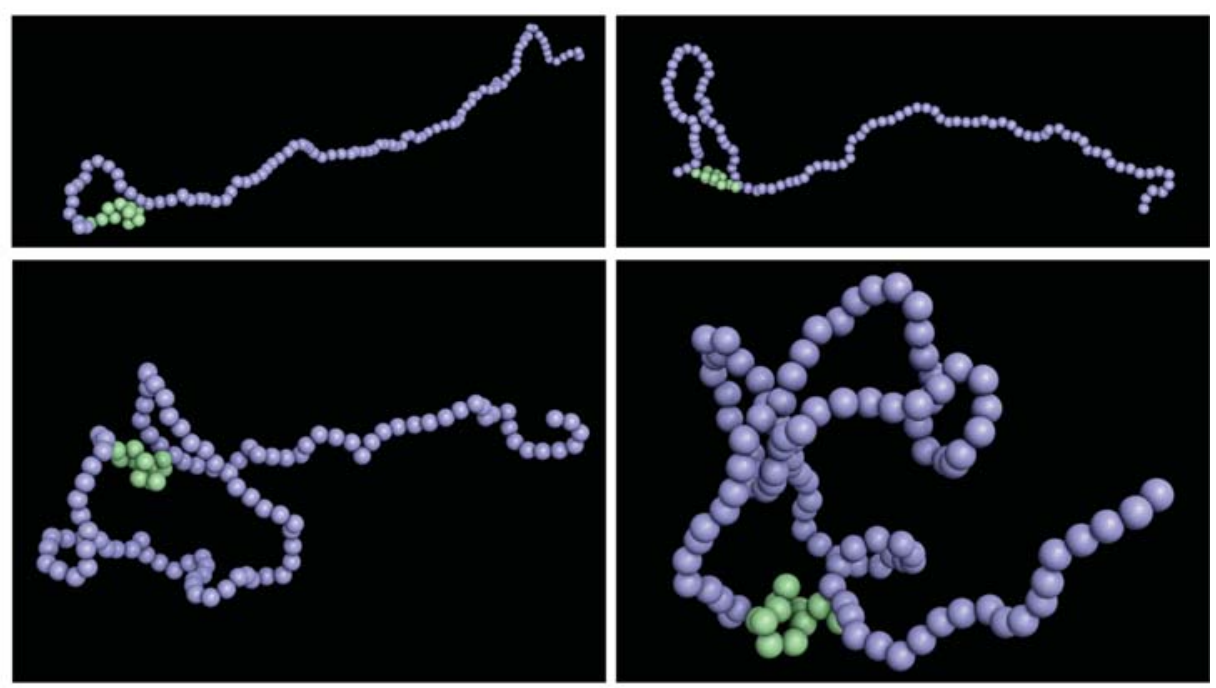

D
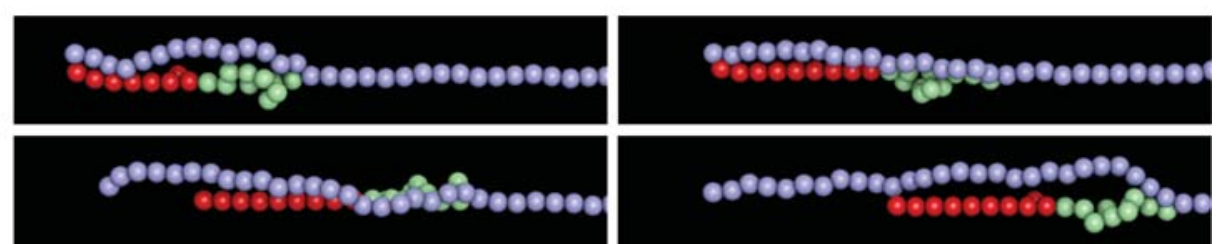

Figure 2. Simulation of condensin walking along a chromatin substrate. (A) Stepping: The DNA (purple beads) with a single condensin (green beads). The DNA ends are tethered to watch how a single condensin molecule steps along the DNA. (B) Looping: The DNA is only tethered at one end, so that its behavior is more like a fluctuating chain in vivo. Note that when condensin steps, loops are extruded as a consequence of the floppiness of the chain. $(C)$ Looping (different camera perspective from $B)$. $(D)$ Translocation: Condensin can bind in cis (the same molecule) or in trans (different molecules). When it binds in trans and takes steps, it will move one DNA strand relative to the other. This is a cross-linking and mobilization function. The two DNA strands are depicted in purple and red. The red strand is fluctuating but is equivalent to the mass of lambda DNA (Terekawa et al. 2017), 10× greater than the mass of the purple beads.

of the floppy strand, which we hypothesized would cause condensin to flip its stepping orientation, a single condensin is able to extrude a loop in a processive manner with simple, proximity-based binding mechanics. This simulation demonstrates loop extrusion is a consequence of processive motion on a floppy substrate. This is an important insight and provides a mechanism for how condensin alters the topology of DNA in vivo to form chromosomes.

Last, Terekawa et al. (2017) reported that condensin is able to proceed along an extended DNA substrate while bound to a separate DNA strand. We replicated this pro- cessive motion of a trans-substrate by placing the Ycs4 beads on a doubly tethered strand and the Ycg1 bead on the other strand. In this situation (Fig. 2D), as condensin walks, the trans-associated molecule is transported along the substrate molecule, recapitulating the phenomena discovered in Terekawa et al. (2017).

\section{Model Convolution}

To translate simulations into experimental images, we implement a method known as model convolution (Gard- 


\section{BOX 1: PARAMETER LIST}

Persistence length of DNA $=50 \mathrm{~nm}$, set by hinge force.

Persistence length of condensin = unset, no hinge force on condensin due to estimates Lp of $4 \mathrm{~nm}$. Two heat-repeat subunit = beads 9 and 10 , condensin to DNA spring strength is same as DNA (2 GPa), rest length $10 \mathrm{~nm}$.

One heat-repeat subunit = bead 1, two binding states, strong state is $2 \mathrm{GPa}$, weak state is $2 \mathrm{MPa}$, rest length is $10 \mathrm{~nm}$, weak state activated when kleisin spring extension is $>30 \mathrm{~nm}(3 \times$ mass separations [thresh in code]).

Kleisin is represented by the distance between beads 2 and 8 . Beads 2 and 8 are joined by bead 11 and two springs (i.e., 2-11-8). Spring strength is $200 \mathrm{MPa}$; rest length of springs are $10 \mathrm{~nm}$. Kleisin threshold is $30 \mathrm{~nm}$.

Step size/rate: Beads 9 and 10 will rotate about each other every $35 \mu \mathrm{sec}$ of simulation time, for one bead to bind to a new DNA bead. Simulations are run at a viscosity of 0.01 P. Given an estimated nuclear viscosity of 141 Poise (Fisher et al. 2009), $35 \mu \mathrm{sec}$ of simulation time is equivalent to $0.5 \mathrm{sec}$. Given that the most common step size is one bead per rotation, this results in a step rate of 2 beads per second. Each bead represents $\sim 30 \mathrm{bp}$, so condensin has a step rate of $60 \mathrm{bp} / \mathrm{sec}$, as described in Terekawa et al. (2017).

A
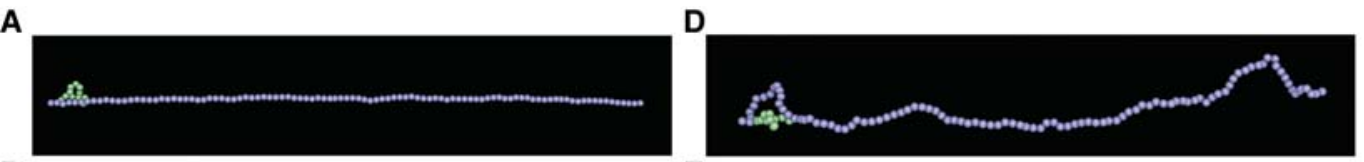

B
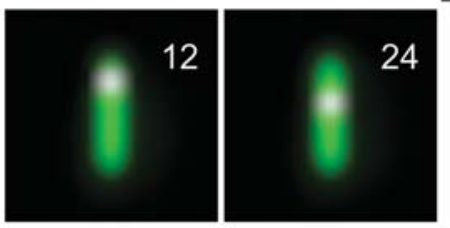

$\mathbf{E}$
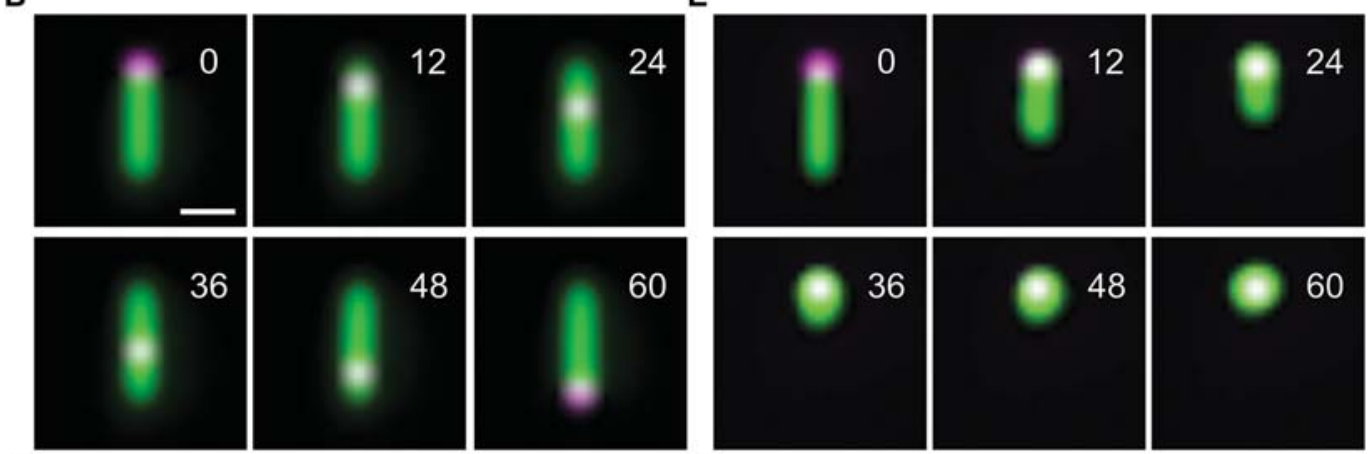

C
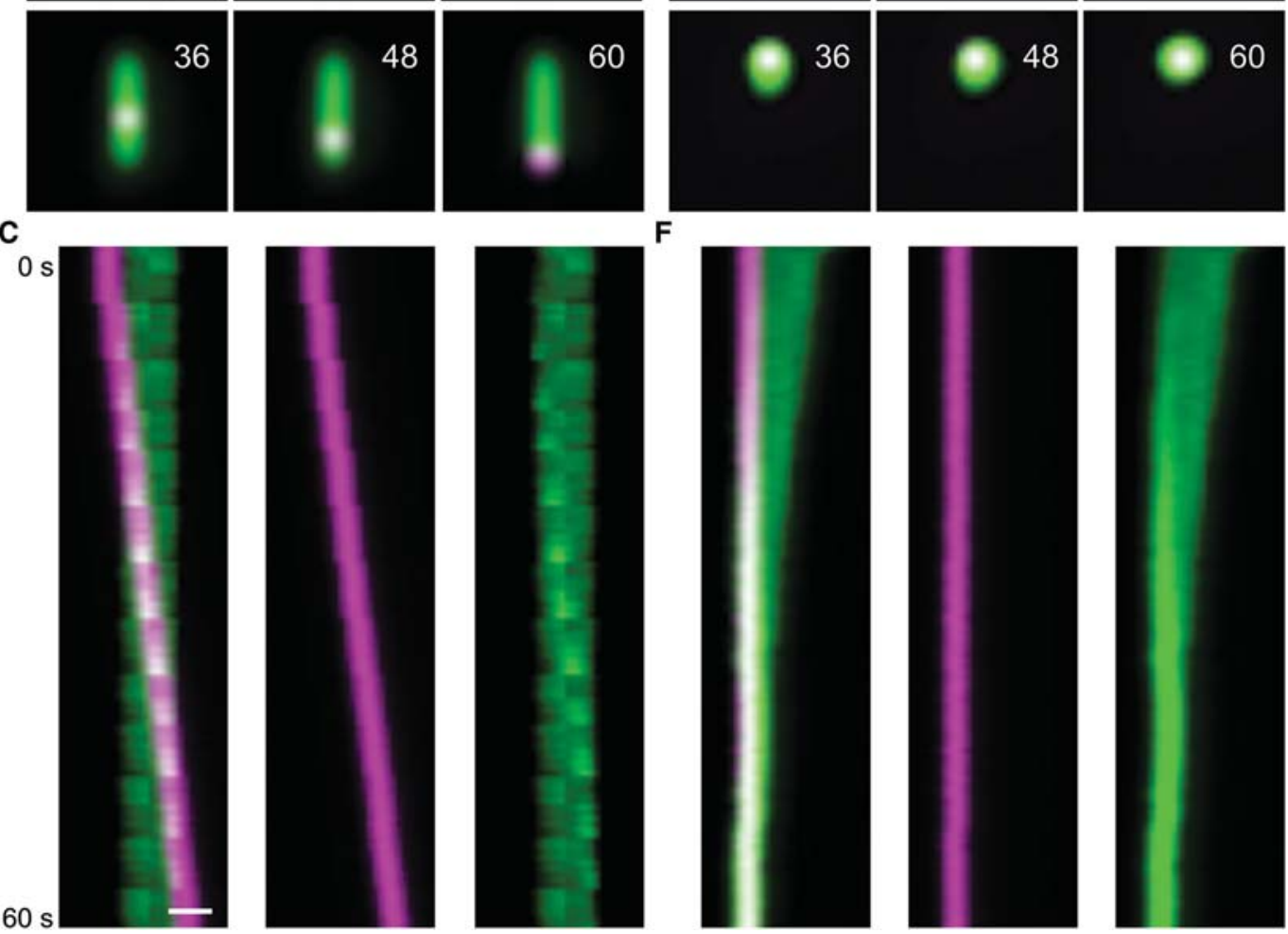

F
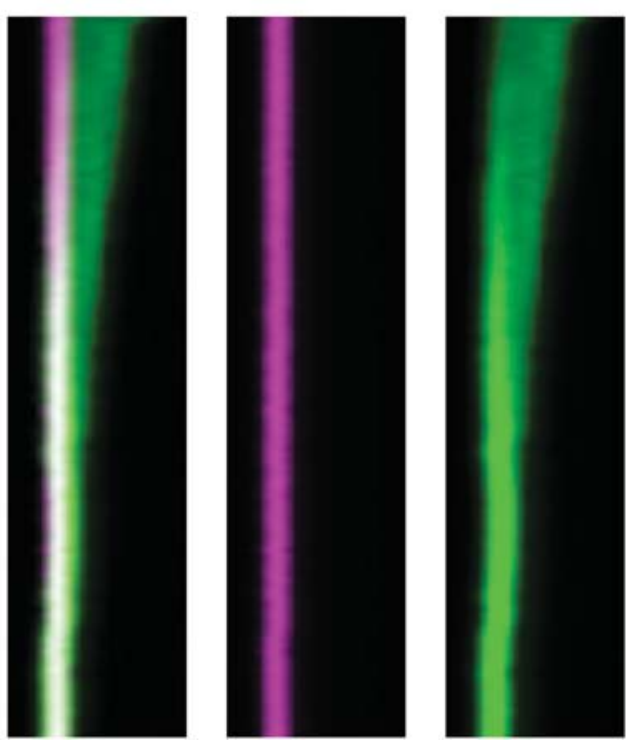

Figure 3. Kymographs of simulated motion convolved through objective point spread function. Rendering of double-tethered DNA $(A)$ and single-tethered DNA $(D)$, simulations containing a single condensin. Condensin is green; DNA is blue. Time series of simulated fluorescence images of double-tethered $(B)$ and single-tethered $(E)$ simulations. Condensin is magenta; DNA is green. Kymographs of double-tethered DNA $(C)$ and single-tethered DNA $(F)$ simulations. Time in seconds. 
ner et al. 2010). We use the microscope objective point spread function to render the simulation into a format that can be directly compared with microscope images of live cells. Figure 3 shows the behavior of condensin walking on a pinned DNA substrate. Note the correspondence between this image and those obtained by Terekawa et al. (2017) for condensin translocating on DNA. The extrusion of DNA loops when condensin translocates along an unpinned substrate readily shows that the DNA compacts into a diffraction-limited spot (Fig. 3E,F). These simulations (Fig. 2) and model convolution (Fig. 3) provide proof of principle that RotoStep can recapitulate the behavior of condensin in vitro.

\section{Strand Compaction via Multiple Condensins}

In vivo, there are multiple condensin molecules on each chromosome. In budding yeast, the density of condensin is about 1 molecule/10 $\mathrm{kb}$ in the bulk of the chromosome and about 1 molecule $/ 3 \mathrm{~kb}$ in the pericentromere. We placed 10 molecules of condensin on a single-tethered chain $(5 \mu \mathrm{m}, 15 \mathrm{~kb})$. The rules for condensin steps are as previously described, and the step orientation for each molecule is random (Fig. 4). As individual condensin's take steps, loops are extruded along the strand (as shown for a single molecule, Fig. 2C,D), and the strand is rapidly compacted into a chromosome structure. Loop extrusion is independent of the direction that condensin steps. With multiple condensin molecules, as multiple loops form, overall chain length shortens. The backbone is fed into the loops via the step function of each molecule. The surprise is that no new features were implemented beyond the simplest of step functions. Compaction is simply the transfer of the backbone chain into extruded loops (Fig. 4).

\section{Tension along the DNA Polymer in a Metaphase Configuration Results in Transient, Pericentric Loops}

Centromere DNA is tethered to microtubules via their kinetochore attachment sites. The enrichment of condensin can be visualized in vivo as a bar or one or two foci along the spindle axis (Bachellier-Bassi et al. 2008; Ste- phens et al. 2011, 2013). To try to gain insight into the thermodynamics governing condensin localization within the pericentromere we provided a dicentric plasmid substrate (two centromeres) for simulation. The actual plasmid is $11.4 \mathrm{~kb}$ ( $\sim 3.86$ micron, 386 beads in simulation). The position of the two centromeres (tethered ends) are indicated as pink beads (Fig. 5) and lie $800 \mathrm{~nm}$ from one another, roughly the distance between separated sister kinetochores in budding yeast metaphase (Pearson et al. 2001). The color of beads on the polymer reflect yeast DNA (blue) and a repeat array of tetracycline operator (white) as described in Lawrimore et al. (2015).

In our simulations, condensin walks along the chain as described above, randomly oriented with respect to one another (Fig. 5). It is difficult for condensin to step through the centromere (simulated as tether sites in pink) because of the geometry of the beads, which increased the dwell time of condensin near the centromeres. Thus, there is kinetic delay for a single condensin molecule at the centromere. This reflects the situation in cells, in which the centromere is at the apex of a stereotypic loop where the kinetochore attaches to microtubules (Yeh et al. 2008). Over time, additional condensin molecules arrive, also extruding loops. The concentration of loops increase and now each condensin can "jump" from one loop to another, amplifying the kinetic delay. The emergent phenomenon is that condensin tends to accumulate near the centromere tether sites. This reflects the density of loops at tether sites. We predict that the accumulation of loops at the centromere reflects the in vivo situation. Because of stochastics of motion in the loops and condensin stepping, a single condensin molecule will escape one position and start toward the other centromere. At this juncture, the loop density decreases, which biases the remaining condensin molecules to "follow" the initial escapee. In silico, this results in condensin molecules "chasing" one another until they accumulate at the other centromere and the cycle continues.

There are several in vivo behaviors that now can be accounted for. Condensin appears as foci that could have been interpreted as oligomerization. The alternative explanation based on simple thermodynamics is that the concentration of condensin reflects the local density of loops. Where loop density is high, condensin will accumulate

A

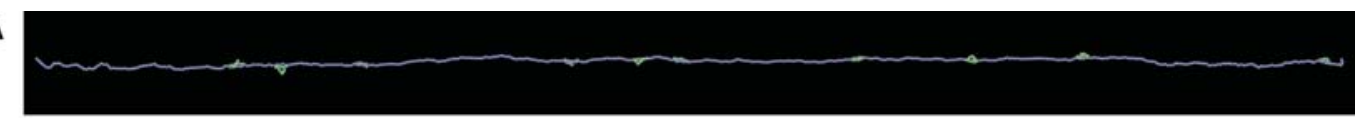

B

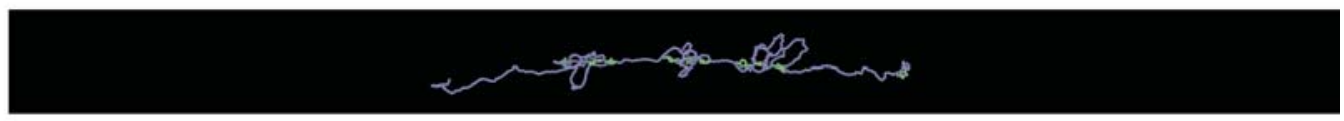

C

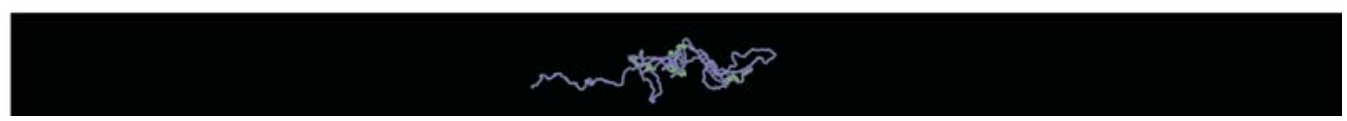

Figure 4. Chromosome compaction. Several condensin molecules were distributed in random position and direction. The DNA molecule is unpinned. As condensin steps $(A-C$, over time), the DNA gets reeled in and the entire ensemble rapidly condenses into a dense aggregate of DNA and condensin. 
A

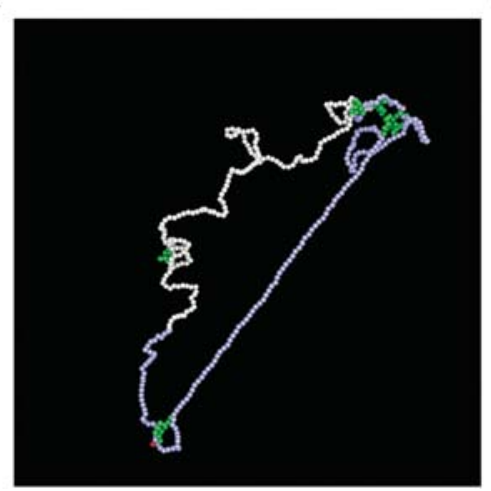

B

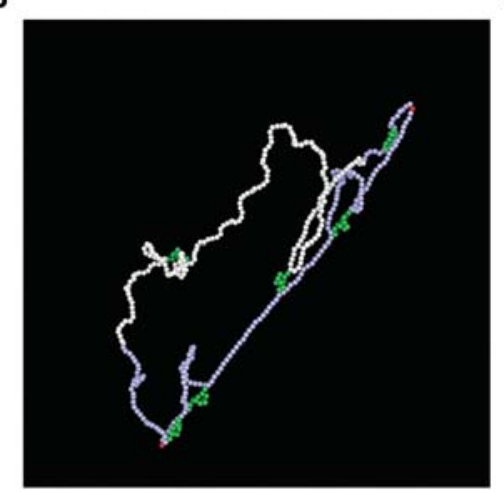

C

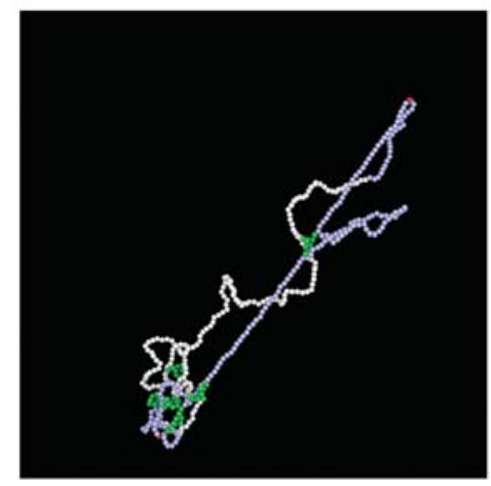

Figure 5. Motion on a dicentric plasmid. A dicentric plasmid in metaphase is simulated by tethering two DNA beads at the position of centromeres within the plasmid (DNA/bead coordinates) and in space at the position of separated kinetochores in yeast metaphase (Lawrimore et al. 2015). The purple beads represent plasmid DNA sequences, the white beads the position of repeat arrays of Tet operator used to visualize with plasmid with TetR-GFP. The number of condensin molecules (green) represents the average density of condensin within the pericentric region of the centromere. Condensin is randomly positioned with respect to DNA sequence and direction. Upon simulated motion $(A-C$, over time), condensin travels from one tether to the other. Condensin aggregates at the tethers, as a function of the number of loops (i.e., beads). Random fluctuations result in individual molecules migrating from one tether to the other. Once one condensin escapes the aggregate, the DNA density decreases (fewer loops, i.e., fewer beads), biasing additional molecules to migrate away. The appearance is that condensins chase one another. This phenocopies what is observed in live cells, although we cannot observe the behavior of single molecules.

because of the increase in substrate concentration. The second behavior is the directional instability observed in vivo. This can be accounted for through stochastics of a single molecule that escapes the region of loops. As one molecule leaves, the loop density is reduced triggering a cascade that will allow it to escape and accumulate on the other side.

\section{Condensin Stepping Creates Condensin-Rich DNA Loops near Tethered Centromeres}

We performed statistical analysis on two dicentric plasmids with the same initial placement of condensin, but with mobile (Fig. 6A,B) and immobile condensin (Fig. $6 \mathrm{C}, \mathrm{D}$ ). The probability distribution of condensin (Fig. 6A,C) and DNA beads (Fig. 6B,D) over all time points of the simulations are shown. In simulations with dynamic condensin there is a greater correlation between condensin number and DNA-strand density than immobile conden$\sin$ (Fig. 6E). Mobile condensin colocalizes with centromeres more often than immobile condensin (Fig. 6F). The enrichment of condensin observed at centromeres may be due to the increased condensin dwell time at tether sites, resulting in the accumulation of centromere-proximal DNA loops. These DNA loops in turn increase the concentration of condensin near centromeres, providing a positive-feedback loop. This pattern of condensin is consistent with that observed in vivo (Lawrimore et al. 2015). With static condensin, there is more condensin along the axis (Fig. 6A,C). Consequently, the DNA has more freedom of movement, which is reflected in the DNA density distribution being radially displaced from the axis (Fig. 6B,D), recapitulating in vivo experiments of the temperature-sensitive condensin mutation brn1-9 (Stephens et al. 2011).
Figure 7 demonstrates that the motion of the substrate confounds the analysis of condensin turnover because of motion of the substrate. Paradoxically, even a highly processive motor does not appear to recover after photobleaching because of a dense, floppy DNA substrate.

\section{CONCLUSION}

We have used a statistical mechanics model of chromatin to examine the consequences of translocation of a motor protein such as condensin on a floppy chromatin substrate. Terekawa et al. (2017) recently reported that condensin is a mechanochemical motor protein. Through simulations that recapitulate in vitro findings we are able to query the consequences of motor stepping on a thermodynamically mobile substrate. Unlike the behavior of motor proteins on DNA sheets under flow, or kinesin-like microtubule-based motor proteins walking on a rigid microtubule, the behavior of the chromatin substrate has a disproportionate contribution to the behavior of a generic DNA-based translocase. Translocation along a floppy substrate is more akin to the random walk of a segment of a fluctuating polymer chain (i.e., subdiffusive Rouse chain [Rubinstein and Colby 2003]). As the translocase steps, the underlying substrate undergoes fluctuations that randomize the direction of the next step (compare Figs. 2 and 3).

At each step, the chromatin is constantly exploring configurations that maximize entropy. In other words, thermal motion is driving the chain toward a random coil. The net consequence is the extrusion of DNA loops (Fig. 2B,C). A key parameter in terms of cell physiology is the stiffness of the chromatin substrate, which can be altered by tethers and the presence of other DNA motors/loop extruders generating regions of taut substrate. If the local substrate is floppy, there will be little if any directed motion. In 


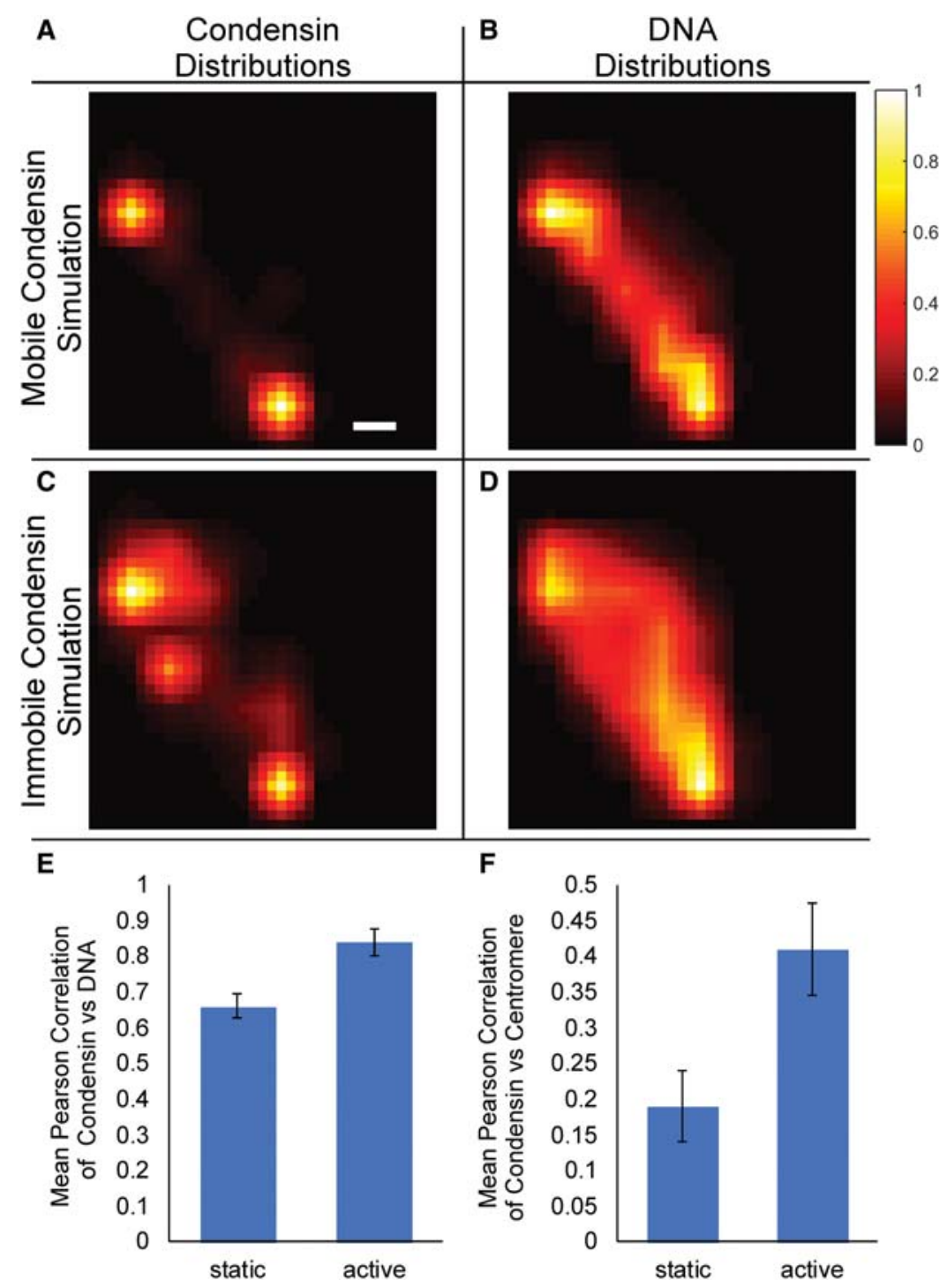

Figure 6. Condensin enrichment at centromere-proximal DNA loops in simulation with mobile vs. immobile condensin. The DNA substrate is the dicentric plasmid described in Figure 5 (both ends pinned at centromeres). Condensin is randomly positioned and oriented along the dicentric molecule. Condensin can step along the DNA in $A, B$, but is static in $C, D$. The starting positions of condensin are the same in both simulations. A probability density map of condensin is shown in $A, C$ and the plasmid DNA in $B, D$. $(E, F)$ Statistical analysis of the correlation of condensin with DNA $(E)$ and centromere (tethered beads) $(F)$. Mobile condensin tends to accumulate at the sites of tethering near the centromeres, resulting in condensin-rich, centromere-proximal DNA loops. DNA tends to show greater radial freedom of motion in simulation with mobile condensin (width of $B$ vs. $D$ ). Scale bar, $100 \mathrm{~nm}$.

contrast, if the substrate is stiffer because of extension, the motor will be more prone to display directed motion. The behavior is also dependent on whether the substrate ends are tethered. If not, even a flexible translocase will enhance the ability of the substrate to adopt a random coil (Fig. 3E). It is reported that condensin is very flexible (Eeftens et al. 2016) (short persistence length, $4 \mathrm{~nm}$ ), and thus may show bursts of directed motion in vivo, depending on the distribution of tethers along the chromosome.

A second feature of translocation along a floppy substrate is compaction. Because there is no vectorial motion, the motor will decrease the time required for the chain to adopt a random coil. This may be perceived as active condensation, but it is a natural consequence of random stepping on a thermally fluctuating chain. Likewise, condensin can appear to concentrate in particular locations, such as within the pericentromere and the nucleolus (Bachellier-Bassi et al. 2008; Stephens et al. 2013; Snider et al. 2014). This has been interpreted as oligomerization between condensin holoenzymes or other cross-linking proteins. The alternative interpretation from the statistical mechanics model is that the concentration of DNA loops increases because of condensin's inability to quickly traverse the centromere region. As condensin steps and extrudes loops, in an environment where there are a plethora 
A
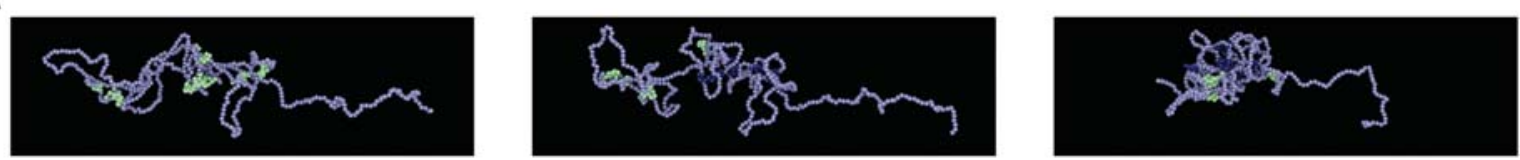

B
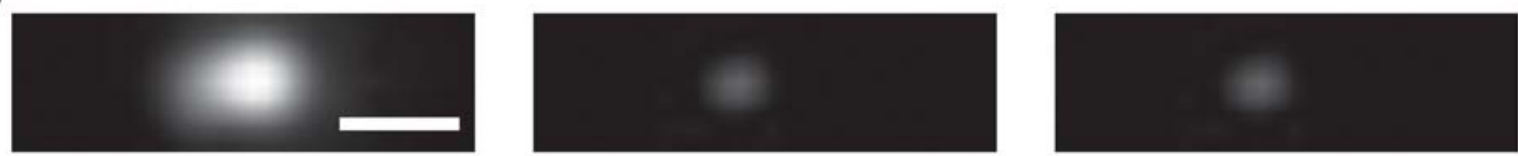

C

1.2

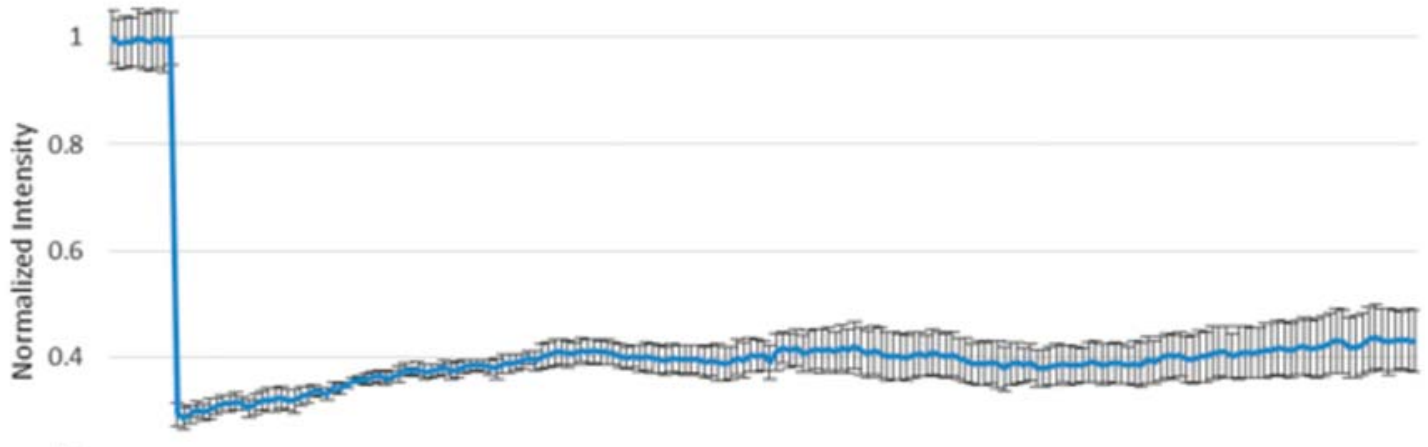

0.2

0

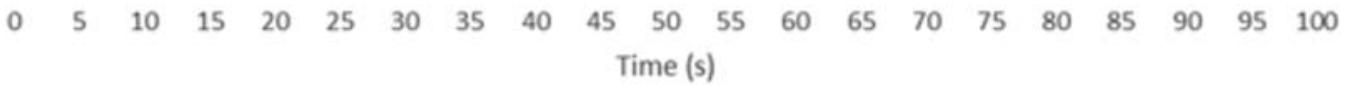

Figure 7. Simulated FRAP experiment by model convolution. Condensin molecules randomly positioned on a 5-kb DNA chain and convolved with the point spread function (PSF) (described above) to simulate a microscopic image. (A) A diffraction limited spot ( 250 micron) was drawn around several condensin molecules. Bleaching was simulated by marking these molecules dark (blue). $(B)$ Fluorescence pre- and postbleach (from left to right). Scale bar, $0.5 \mu \mathrm{m} .(C)$ Mean normalized quantitative fluorescence recovery. Curve is mean of three normalized recovery curves. Error bars are standard error of the mean. The finding in simulation is that any recovery can be attributed to fluctuation of the chain (and associated condensin) into the bleach zone. Thus FRAP is confounded by the thermodynamics of the substrate. (Compare $B$ to Fig. 5 in Lawrimore et al. [2015].)

of molecules, condensin on one loop can readily step to a physically adjacent loop. This is evident in Figure 6, showing accumulation of condensin at sites of tethers, corresponding with increased concentration of DNA loops. Thus, perceived condensin accumulation may reflect the propensity for loops to congregate condensin, which produces more loops. Condensin's ability to step from one loop to another provides a positive-feedback mechanism to increase the duration of these transient events and the local concentration of DNA loops. However, the stochastics of the system will result in occasional molecules escaping sites of loop accumulation by traversing the taut regions of the substrate. As shown in Figure 5, when one molecule escapes, the loop density decreases, thus biasing additional molecules to exit sites of loop accumulation. This unstable positive-feedback loop may contribute to condensin's enrichment at the pericentromere and nucleolus in vivo. Such a transient, positive feedback may explain the existence of TADs in population studies, but not in single cells (Flyamer et al. 2017). In essence, the organization of the DNA biases the occasional enrichment of condensin based on the presence of a tether causing a loop.

The statistical mechanics of polymer behavior and protein translocation provides a powerful tool to build intuition for understanding experimental observation and making physically accurate hypotheses. Using the simple assumptions of proximity-based substrate binding and extensional-dependent substrate unbinding, we provide alternative explanations for several experimental observations and make new predictions about the rules for loop persistence and extrusion. The model highlights potential new functions for tethers along the chromosome and how the distribution of tethers will be intimately involved in loop formation at a distance (Fig. 3B,E). Finally, the mechanical feedback between loops in the same proximity provides new perspectives on interchromosomal communication. 


\section{ACKNOWLEDGMENTS}

We thank Dr. Paula Vasquez (University of South Carolina) and Dr. Elaine Yeh (University of North Carolina, Chapel Hill) for discussion and critical reading of the manuscript. This work was supported by the National Institutes of Health General Medicine, R37GM32238 to K.B. and T32CA201159-01 and T32GM007092-39 to J.L.

\section{REFERENCES}

Alberts BM, Barry J, Bedinger P, Formosa T, Jongeneel CV, Kreuzer KN. 1983. Studies on DNA replication in the bacteriophage T4 in vitro system. Cold Spring Harb Symp Quant Biol 47 Pt 2: 655-668.

Alipour E, Marko JF. 2012. Self-organization of domain structures by DNA-loop-extruding enzymes. Nucleic Acids Res 40: 11202-11212.

Bachellier-Bassi S, Gadal O, Bourout G, Nehrbass U. 2008. Cell cycle-dependent kinetochore localization of condensin complex in Saccharomyces cerevisiae. J Struct Biol 162: 248-259.

Dekker J, Marti-Renom MA, Mirny LA. 2013. Exploring the three-dimensional organization of genomes: Interpreting chromatin interaction data. Nat Rev Genet 14: 390-403.

Dostie J, Bickmore WA. 2012. Chromosome organization in the nucleus - Charting new territory across the Hi-Cs. Curr Opin Genet Dev 22: 125-131.

Earnshaw WC, Halligan B, Cooke CA, Heck MM, Liu LF. 1985. Topoisomerase II is a structural component of mitotic chromosome scaffolds. J Cell Biol 100: 1706-1715.

Eeftens JM, Katan AJ, Kschonsak M, Hassler M, de Wilde L, Dief EM, Haering CH, Dekker C. 2016. Condensin Smc2-Smc4 dimers are flexible and dynamic. Cell Rep 14: 1813-1818.

Fazio T, Visnapuu ML, Wind S, Greene EC. 2008. DNA curtains and nanoscale curtain rods: High-throughput tools for single molecule imaging. Langmuir 24: 10524-10531.

Fisher JK, Ballenger M, O'Brien ET, Haase J, Superfine R, Bloom K. 2009. DNA relaxation dynamics as a probe for the intracellular environment. Proc Natl Acad Sci 106: 9250-9255.

Flyamer IM, Gassler J, Imakaev M, Brandao HB, Ulianov SV, Abdennur N, Razin SV, Mirny LA, Tachibana-Konwalski K. 2017. Single-nucleus Hi-C reveals unique chromatin reorganization at oocyte-to-zygote transition. Nature 544: 110-114.

Gardner MK, Sprague BL, Pearson CG, Cosgrove BD, Bicek AD, Bloom K, Salmon ED, Odde DJ. 2010. Model convolution: A computational approach to digital image interpretation. Cell Mol Bioeng 3: 163-170.

Hertwig O. 1906. Lehrbuch der Entwicklungsgeschichte des Menschen und der Wirbeltiere [Textbook of developmental history of humans and vertebrates]. Reissue 2016. Wentworth Press, Sydney.

Hirano T. 2006. At the heart of the chromosome: SMC proteins in action. Nat Rev Mol Cell Biol 7: 311-322.
Kull FJ, Sablin EP, Lau R, Fletterick RJ, Vale RD. 1996. Crystal structure of the kinesin motor domain reveals a structural similarity to myosin. Nature 380: $550-555$.

Lawrimore J, Vasquez PA, Falvo MR, Taylor RM II, Vicci L, Yeh E, Forest MG, Bloom K. 2015. DNA loops generate intracentromere tension in mitosis. J Cell Biol 210: 553-564.

Lawrimore J, Aicher JK, Hahn P, Fulp A, Kompa B, Vicci L, Falvo M, Taylor RM II, Bloom K. 2016. ChromoShake: A chromosome dynamics simulator reveals that chromatin loops stiffen centromeric chromatin. Mol Biol Cell 27: 153166.

Ou HD, Phan S, Deerinck TJ, Thor A, Ellisman MH, O'Shea CC. 2017. ChromEMT: Visualizing 3D chromatin structure and compaction in interphase and mitotic cells. Science 357: 370-382.

Park K, Debyser Z, Tabor S, Richardson CC, Griffith JD. 1998. Formation of a DNA loop at the replication fork generated by bacteriophage $\mathrm{T} 7$ replication proteins. J Biol Chem 273: 52605270.

Paulson JR, Laemmli UK. 1977. The structure of histone-depleted metaphase chromosomes. Cell 12: 817-828.

Pearson CG, Maddox PS, Salmon ED, Bloom K. 2001. Budding yeast chromosome structure and dynamics during mitosis. $J$ Cell Biol 152: 1255-1266.

Piazza I, Rutkowska A, Ori A, Walczak M, Metz J, Pelechano V, Beck M, Haering CH. 2014. Association of condensin with chromosomes depends on DNA binding by its HEAT-repeat subunits. Nat Struct Mol Biol 21: 560-568.

Rubinstein M, Colby RH. 2003. Polymer physics. Oxford University Press, Oxford.

Sinha NK, Morris CF, Alberts BM. 1980. Efficient in vitro replication of double-stranded DNA templates by a purified T4 bacteriophage replication system. J Biol Chem 255: 42904293.

Snider CE, Stephens AD, Kirkland JG, Hamdani O, Kamakaka RT, Bloom K. 2014. Dyskerin, tRNA genes, and condensin tether pericentric chromatin to the spindle axis in mitosis. $J$ Cell Biol 207: 189-199.

Stephens AD, Haase J, Vicci L, Taylor RM II, Bloom K. 2011. Cohesin, condensin, and the intramolecular centromere loop together generate the mitotic chromatin spring. JCell Biol 193: $1167-1180$.

Stephens AD, Quammen CW, Chang B, Haase J, Taylor RM II, Bloom K. 2013. The spatial segregation of pericentric cohesin and condensin in the mitotic spindle. Mol Biol Cell 24: 3909 3919.

Terekawa T, Bisht S, Eeftens JM, Dekker C, Haering CH, Greene EC. 2017. The condensin complex is a mechanochemical motor that translocates along DNA. Science 358: 672-676.

Vasquez PA, Hult C, Adalsteinsson D, Lawrimore J, Forest MG, Bloom K. 2016. Entropy gives rise to topologically associating domains. Nucleic Acids Res 44: 5540-5549.

Yeh E, Haase J, Paliulis LV, Joglekar A, Bond L, Bouck D, Salmon ED, Bloom KS. 2008. Pericentric chromatin is organized into an intramolecular loop in mitosis. Curr Biol 18: $81-90$. 


\section{$\$_{\text {CSH }}^{\infty}$ Cold Spring Harbor Symposia SYMPOSIA On Quantitative Biology}

\section{RotoStep: A Chromosome Dynamics Simulator Reveals Mechanisms of Loop Extrusion}

Josh Lawrimore, Brandon Friedman, Ayush Doshi, et al.

Cold Spring Harb Symp Quant Biol 2017 82: 101-109 originally published online November 22, 2017

Access the most recent version at doi:10.1101/sqb.2017.82.033696

References This article cites 27 articles, 12 of which can be accessed free at: http://symposium.cshlp.org/content/82/101.full.html\#ref-list-1

Creative This article is distributed under the terms of the

Commons http://creativecommons.org/licenses/by-nc/4.0/, which permits reuse and

License redistribution, except for commercial purposes, provided that the original author and source are credited.

Email Alerting Receive free email alerts when new articles cite this article - sign up in Service the box at the top right corner of the article or click here. 